$\mathrm{Zu}$ den chronischen Erkrankungen, die ganzheitlich praktizierende Ärzte am häufigsten sehen, gehört die Migräne. Jede fünfte Frau und jeder siebte Mann leidet unter ihr. In der Schweiz sind nach einer Schätzung der Schweizerischen Kopfwehgesellschaft eine Million Menschen durch die Migräne betroffen. Sie wird von der Weltgesundheitsorganisation (WHO) als eine der am meisten einschneidenden Beeinträchtigungen des Lebens eingestuft. Für die Betroffenen ist die Migräne oft eine grosse Last. Die Symptome von Kopfschmerzen über Augenflimmern bis hin zu neurologischen Ausfällen machen während des Anfalls ein normales Leben unmöglich und beeinträchtigen so die Lebensqualität stark. Viele leiden über Jahre an den immer wieder auftretenden Episoden und suchen, wenn die Selbstmedikation und der Hausarzt oder Neurologe keinen Erfolg bringen, bei der ganzheitlichen Medizin Hilfe. Etwa 30\% der Betroffenen gehen diesen Weg. Während das phytotherapeutische Arsenal für die Behandlung der Migräne gut bestückt ist, herrscht Unklarheit, welche anderen Ansätze der Ganzheitsmedizin verlässlich wirken. Oft sind die Ergebnisse verschiedener wissenschaftlicher Studien widersprüchlich. So gibt es etwa Hinweise, dass die Akupunktur wirkt, sich allerdings bei der Wirkung von richtiger und Scheinakupunktur kein Unterschied finden lässt. Im vergangenen Jahr ist eine Reihe von Studien erschienen, die den Einsatz der Homöopathie bei der Prophylaxe der Migräne untersuchen. Sie ist Teil einer grösseren Untersuchung an der Charité in Berlin, die sich mit der Wirkung der Homöopathie bei verschiedenen Krankheiten beschäftigt. In der prospektiven Studie [1] haben Claudia Witt und ihre Mitarbeiter den Behandlungsansatz der Homöopathie bei 212 Patienten, die bei 62 Ärzten in Behandlung waren, ausgewertet. Diese hatten alle

\title{
Mit kleiner Dosis gegen grosse Schmerzen
}

Bei Migräne setzen Komplementärmediziner vor allem auf die Phytotherapie. Hinweise, dass auch die Homöopathie wirkt, eröffnen neue Perspektiven.

eine Ausbildung in Klassischer Homöopathie und praktizierten diesen Ansatz auch konsequent. Das Team der Charité liess die Patienten Fragebögen über die Stärke und Häufigkeit der Migräneanfälle sowie über ihre Lebensqualität vor und während der Behandlung ausfüllen. Die Ergebnisse zeigen, dass sich mit Homöopathie gute Erfolge bei der Behandlung von Patienten mit Migräne erzielen lassen. Im Verlauf der 24 Monate laufenden Studie nahm bei den meisten Betroffenen die Schwere der Migränesymptome $a b$ und die Lebensqualität verbesserte sich. Der grösste Effekt liess sich innerhalb der ersten 3 Monate beobachten. Die Verbesserung setzte sich während der gesamten weiteren Behandlungsdauer weiter fort. Die Behandlung dauerte im Durchschnitt 12 Monate; 6 Verschreibungen waren notwendig. Je besser es Patienten bei der Homöopathiebehandlung ging, desto mehr verringerte sich der Einsatz von anderen Medikamenten. Sie benötigten auch deutlich weniger ärztliche Hilfe. Die Autoren der Studie lassen offen, ob wegen des Erfolgs der Homöopathie weniger andere Medikamente eingenommen werden oder ob die Verschreibungspraxis der behandelnden homöopathischen Ärzte damit zu tun haben könnte. Diese würden den schulmedizinischen Ansatz während ihrer Behandlung eher zurückfahren. Da in der Homöopathie keine standardisierten Produkte, sondern individualisierte Rezepturen verwendet werden, kann man keine Aussage über die Wirkung bestimmter Präparate bei der Migräne machen. 10 homöopathische Substanzen wurden bei etwa der Hälfte aller Patienten eingesetzt. Insgesamt kamen 168 verschiedene Substanzen zur Anwen- dung. Die Studie passt gut zu früheren Untersuchungen, die bereits eine Wirksamkeit der Homöopathie bei der Prophylaxe der Migräne wahrscheinlich gemacht haben. Beispielsweise konnte eine Wirkung gefunden werden, die bei einer «richtigen» Homöopathie höher war als bei einer Scheinbehandlung [2]. Der Erfolg ist eine Reduktion der mittleren und schweren Attacken um etwa ein Drittel [3]. Damit zeigt sich, dass Komplementärmediziner mit der Homöopathie eine weitere ganzheitliche Methode zur Verfügung haben, um Migräne zu behandeln. Als Akuttherapie, um eine akute Episode zu lindern, eignet sie sich allerdings nicht. Hier bleiben die aus der Phytotherapie bewährten Präparate, etwa zehnprozentiges Pfefferminzöl, das so effizient ist wie $1 \mathrm{~g}$ Paracetamol. Die Kombination von Paracetamol und Pfefferminzöl scheint noch besser zu wirken. Um die Anzahl und Schwere der Migräneattacken zu lindern, steht z.B. der Pfefferextrakt Capsaicin zur Verfügung. Die Stärke der Anfälle lindert auch ein Weidenrindenextrakt, das nicht über die SalicinKette wirkt und deshalb darm- und magenschonend ist. Für die Prävention ist das Mutterkraut mit seinem Wirkstoff ideal, und auch PestwurzExtrakte bieten sich an. (rfi)

\section{Literatur}

1 Witt CM, Lüdtke R, Willich SN: Homeopathic treatment of patients with migraine: a prospective observational study with a 2-year follow-up period. J Alt Compl Med 2010;16:347-355.

$\checkmark 2$ Whitmarsh TE, Coleston-Shields DM, Steiner TJ: Double-blind randomised placebocontrolled study of homoeopathic prophylaxis of migraine. Cephalalgia 1997;17:600-604.

3 Straumsheim P, Borchgrevink C, Mowinckel P, et al.: Homeopathic treatment of migraine: a double-blind, placebo-controlled trial of 68 patients. Br Hom J 2000;89:4-7. 